\title{
Control Strategies in Multi-Player Pursuit and Evasion Game*
}

\author{
Jung Soon Jang ${ }^{\dagger}$ and Claire J. Tomlin ${ }^{\ddagger}$ \\ Department of Aeronautics and Astronautics \\ Stanford University, Stanford, CA 94305-4035
}

\begin{abstract}
This paper presents a new methodology for solving multi-player pursuit and evasion games. The proposed control strategies are derived from direct differentiation of chosen value (or level set) functions, instead of solving the associated Hamilton Jacobi Isaacs (HJI) equations. The corresponding strategies offer a simple form of the control laws that can be implemented on real-time control systems for autonomous vehicles. In order to guarantee a minimum separation distance between players, a new method to compute a reachable set, which only requires a set of ordinary differential equations, is developed. We also explore different value functions which lead to different performance measures for some players. We illustrate our approach on a three player pursuit and evasion game and present simulation results.
\end{abstract}

\section{Introduction}

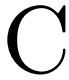
oordination and control of multiple agents have received great attention over the last few years. ${ }^{1-5}$ Applications abound: formation flying, conflict resolution, optimum task allocation, and air-traffic control, just to name a few.

Differential game theories have been studied for obtaining optimal solutions of such problems. Also, advances in numerical methods for partial differential equations (PDEs) have opened an alternative route to study these problems by numerically solving the associated Hamilton-Jocobi equations. Examples have included various two-player games ${ }^{6-8}$ and the associated conflict resolution problem ${ }^{9}$ and multi-player games (more than two). ${ }^{10-12}$ Control strategies presented in the above studies used the knowledge of the exact or approximate solutions of associated HJ equations or of (assumed) opposing intents of some players, in order to devise the corresponding control laws for the players.

While these approaches are mathematically elegant and powerful (a way of capturing the behavior of entire groups of trajectories at once), they suffer from the curse of dimensionality. This not only limits their applicability to low-dimensional problems, but also results in failure of real-time implementation (because of computational complexity and storage). Alternatively, to mitigate these shortcomings, over-approximation schemes have been studied by Mitchell and Tomlin, ${ }^{13}$ Stipanović, Hwang, Tomlin, ${ }^{14}$ and others, ${ }^{15-17}$ and these approximations may be used for fast computations of control laws. Stipanović, Shankaran, and Tom$\operatorname{lin}^{18}$ also presented strategies for multi-player game derived from an approximate Hamilton Jacobi Isaacs (HJI) equation by minimizing or maximizing the growth of chosen level-set functions.

In this paper, we present a solution methodology for multi-player (multi-pursuer) pursuit and evasion game. Control strategies for the players are derived from certain value functions, rather than computing control strategies from the solution of associated HJ equations. The elegance of this method is in that it provides analytical expressions for the (non-optimal) strategies, accommodates nonlinear dynamics easily, and can be extended to include more players and/or different value (or objective) functions. The simplified form of the strategies also offers practical implementation for real-time systems.

\footnotetext{
* This study is supported by the DoD Multidisciplinary University Research Initiative (MURI) program administered by the Office of Naval Research under Grant N00014-02-1-0720.

${ }^{\dagger}$ Research Associate, Department of Aeronautics and Astronautics, AIAA member, jsjang@stanford.edu

$¥$ Associate Professor in Department of Aeronautics and Astronautics, and Courtesy Associate Professor in Department of Electrical Engineering, Director of Hybrid Systems Lab., AIAA member, tomlin@stanford.edu
} 


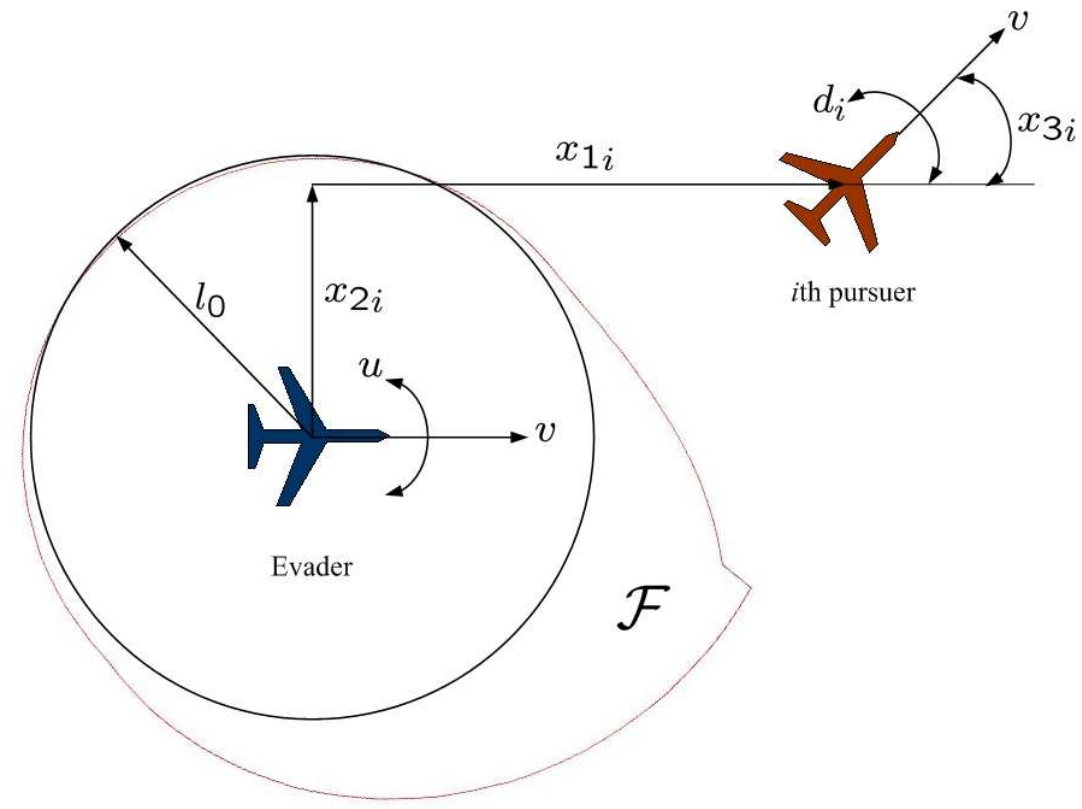

Figure 1. Relative coordinate system: origin is located at the center of the evader, and is oriented so that evader has 0 degree heading angle with respect to the horizontal axis.

This paper is organized as follows. Section II defines the problem discussed in this paper. Section III derives control strategies for players using chosen value functions and computes the corresponding reachable set incorporated into the devised control strategies. Section IV presents simulation results and Section V presents a control strategy for the pursuers derived from a different value function. Finally, Section VI concludes this paper.

\section{Problem Statement}

This section defines some notions for pursuit-evasion game between $n$ identical players. The game is assumed to be of non-zero sum variety. Consider a planar $n$ player game where aircraft dynamics are given in the following relative coordinates (see Figure 1):

$$
\dot{x}_{i}=\frac{d}{d t}\left[\begin{array}{c}
x_{1 i} \\
x_{2 i} \\
x_{3 i}
\end{array}\right]=\left[\begin{array}{c}
-v+v \cos x_{3 i}+u x_{2 i} \\
v \sin x_{3 i}-u x_{1 i} \\
d_{i}-u
\end{array}\right]=f\left(x_{i}, u, d_{i}\right)
$$

where $\left(x_{1 i}, x_{2 i}\right)$ and $x_{3 i}$ respectively represent a relative position and a relative heading angle of the $i$ th pursuer with respect the evader, $v$ is the speed of the aircraft, and $d_{i}$ and $u$ are the control input of the $i$ th pursuer and the evader respectively. Magnitudes of the control input belong to the following norm bounded sets

$$
u \in\{a \in \mathbb{R} \mid\|a\| \leq \mu\}, d_{i} \in\{b \in \mathbb{R} \mid\|b\| \leq \nu\}
$$

where $\mu$ and $\nu$ are positive constants.

Instead of formulating the problem as the corresponding minmax optimization (the pursuer wants to minimize the distance with the evader and the evader wants to maximize it), where the solution is associated with the corresponding HJI equations, the objective of the game is to 1) find control strategies satisfying the following constraint:

$$
\begin{aligned}
& \left\{\begin{aligned}
u^{*} & \rightarrow \dot{J}_{e}>0 \\
d_{i}^{*} \rightarrow \dot{J}_{p_{i}} & <0
\end{aligned}\right. \\
& \text { subject to }\|u\| \leq \mu,\left\|d_{i}\right\| \leq \nu
\end{aligned}
$$

$$
2 \text { of } 10
$$


where $J_{e}=\sum_{i=1}^{n} J_{p_{i}}$ and $J_{p_{i}}=x_{1}^{2}+x_{2}^{2}$ are value functions associated with the evader and individual pursuers respectively. This implies that the pursuer wants to keep decreasing its distance from the evader and the evader wants to increase the sum of distances with all pursuers. However, the proposed evader's control strategy does not necessarily guarantee that the evader can avoid capture by any pursuer since the evader's constraint $\left(\dot{J}_{e}>0\right)$ can be easily satisfied: for example, $\dot{J}_{e}=\sum_{i=1}^{n} \dot{J}_{p_{i}}>0$ is still valid even with $\dot{J}_{p_{i}}<0, i=k+1, \ldots, n$, if $\sum_{i=1}^{k} \dot{J}_{p_{i}}>\sum_{i=k+1}^{n} \dot{J}_{p_{i}}$. To prevent this from happening, we also would like to 2) compute a reachable set $\mathcal{F}$ such that if the evader applies the proposed control strategy on the boundary of this set, $\partial \mathcal{F}$, then none of pursuers can enter a given target set (in Figure 1, this target set is denoted by the disk around the evader of radius $l_{0}$ ).

\section{A Game of Identical Vehicles}

This section derives control strategies for both evader and pursuers using the value functions defined in the previous section and computes the corresponding forward reachable set that the evader can use to keep a minimum separation distance from pursuers.

\section{A. Derivation of Control Strategy}

The proposed control strategies are obtained by differentiating the value functions associated with each player. The time derivative of $J_{p_{i}}$ associated with the $i$ th pursuer is given by

$$
\begin{aligned}
\dot{J}_{p_{i}} & =2 \dot{x_{1 i}} x_{1 i}+2 \dot{x_{2 i}} x_{2 i} \\
& =2\left(-v+v \cos x_{3 i}+u x_{2 i}\right) x_{1 i}+2\left(v \sin x_{3 i}-u x_{1 i}\right) x_{2 i} \\
& =2 v\left[\left(\cos x_{3 i}-1\right) x_{1 i}+x_{2 i} \sin x_{3 i}\right]
\end{aligned}
$$

However, the result is not an explicit function of control inputs, but we may keep differentiating the value function until control inputs appear (as we do in feedback linearization or dynamic extension). As a result, the second time derivative of $J_{p_{i}}$ is given by

$$
\begin{aligned}
\ddot{J}_{p_{i}} & =2 v\left\{-\sin x_{3 i} \dot{x_{3 i}} x_{1 i}+\left(\cos x_{3 i}-1\right) \dot{x_{1 i}}+\cos x_{3 i} \dot{x_{3 i}} x_{2 i}+\sin x_{3 i} \dot{x_{2 i}}\right\} \\
& =\left(x_{2 i} \cos x_{3 i}-x_{1 i} \sin x_{3 i}\right) d_{i}-x_{2 i} u+2 v\left(1-\cos x_{3 i}\right)
\end{aligned}
$$

Similarly, the second time derivative of the evader's value function $J_{e}$ is given by

$$
\begin{aligned}
\ddot{J}_{e} & =\sum_{i=1}^{n} \ddot{J}_{p_{i}} \\
& =-u \sum_{i=1}^{n} x_{2 i}+\sum_{i=1}^{n}\left\{\left(x_{2 i} \cos x_{3 i}-x_{1 i} \sin x_{3 i}\right) d_{i}+2 v\left(1-\cos x_{3 i}\right)\right\}
\end{aligned}
$$

Using (5) and (6), we can reconstruct control strategies to satisfy the following minmax problem:

$$
\begin{aligned}
d_{i}^{*} & \rightarrow \min _{\left\|d_{i}\right\| \leq \nu} \ddot{J}_{p_{i}} \\
u^{*} & \rightarrow \max _{\|u\| \leq \mu} \ddot{J}_{e}=\max _{\|u\| \leq \mu}\left(\sum_{i=1}^{n} \min _{\left\|d_{i}\right\| \leq \nu} \ddot{J}_{p_{i}}\right)
\end{aligned}
$$

and the corresponding solution is given by

$$
\begin{aligned}
d_{i}^{*} & =-\operatorname{sign}\left(x_{2 i} \cos x_{3 i}-x_{1 i} \sin x_{3 i}\right) \nu \\
u^{*} & =-\operatorname{sign}\left(\sum_{i=1}^{n} x_{2 i}\right) \mu
\end{aligned}
$$

The above control strategies do not necessarily imply that the original constraint (3) is satisfied throughout the game, since $d_{i}^{*}$ and $u^{*}$ are bounded and the sign of $\dot{J}_{p_{i}}$ and $\dot{J}_{e}$ are dependent upon initial values of

$$
3 \text { of } 10
$$




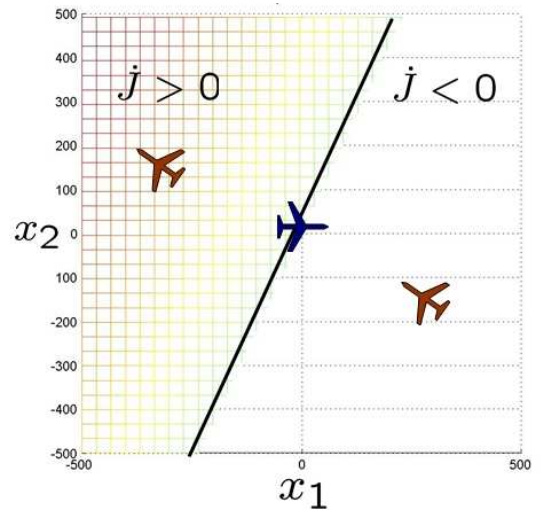

(a) A plot of $\dot{J}(0)$ at $x_{3}(0)=130$ degrees.

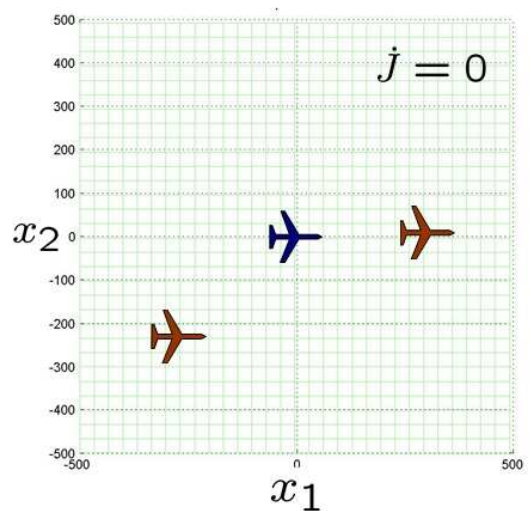

(b) A plot of $\dot{J}(0)$ at $x_{3}(0)=0$ degrees.

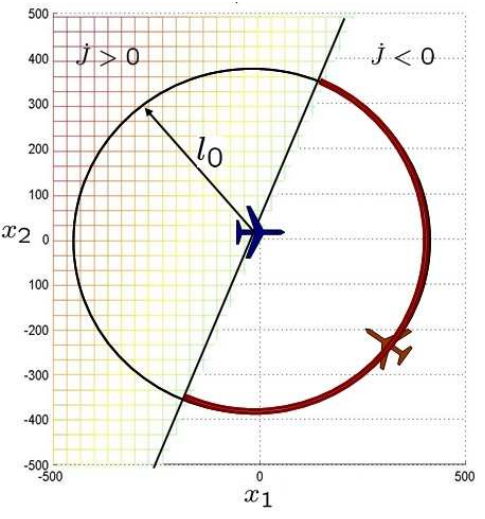

(c) A plot of usable part at a given $x_{3}(0)=130$ degrees.

Figure 2. A plot of $\dot{J}(0)$ over $x_{1}$ and $x_{2}$ axes at given relative heading angles, for two player game.

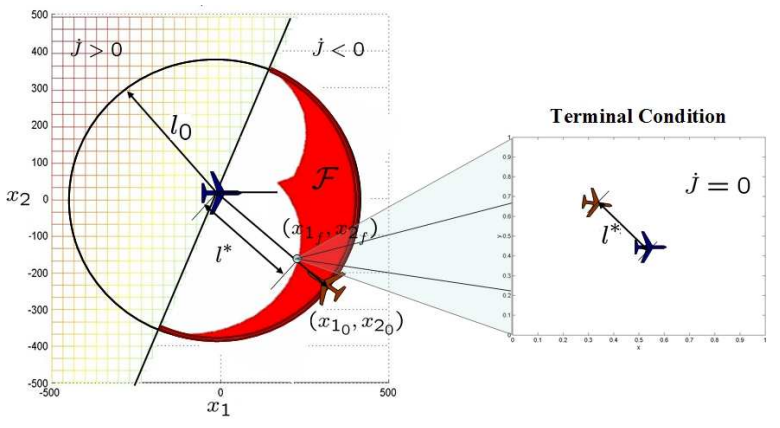

(a) A forward reachable set at $x_{3}=130$ degrees.

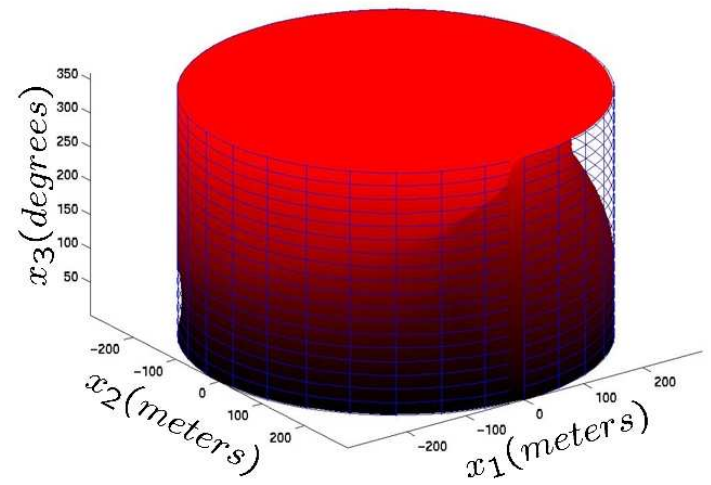

(b) A complete forward reachable set.

Figure 3. A plot of the forward reachable set for two player game.

$\dot{J}_{p_{i}}(0)$ and $\dot{J}_{e}(0)$. In other words, the game is heavily dependent upon initial configurations since all players are equally capable. For example, Figure 2 shows a plot of $\dot{J}(0)\left(=\dot{J}_{e}=\dot{J}_{p}\right)$ for a two player game, at a given relative heading angle. Here, the evader is depicted at the origin, and two different cases of initial configuration are displayed for a pursuer. As seen in Figure 2(a), depending on the relative position of the pursuer with respect to the evader, either the pursuer $(\dot{J}(0)<0)$ or the evader $(\dot{J}(0)>0)$ may take advantage of a given initial configuration. $\dot{J}(t)=0$ is only possible when the relative heading angle is zero, meaning that neither the pursuer nor the evader can change their relative distance. That is, the game reaches the steady state.

Therefore, in order to guarantee that the evader can maintain a minimum distance from the pursuers even at initial configurations of $\dot{J}_{e}(0)<0$, we need to find a reachable space which partitions "capture" from "evade". This is discussed in the next section.

\section{B. Computing the Reachable Set for Continuous Dynamics}

This section, for simplicity, starts with a two player game to explain how to compute a forward reachable set and presents the idea of "reflection" of the reachable set. The corresponding results can be easily extended to multi-player games.

Consider a case in which the pursuer can be on any location of the circle defining the boundary of the target set at a given initial relative heading angle (see Figure $2(\mathrm{c})$ ). The pursuer on the semi-circle defined by $\dot{J}(0)<0$ can move closer to the evader. Therefore this section becomes a usable part for the reachable 


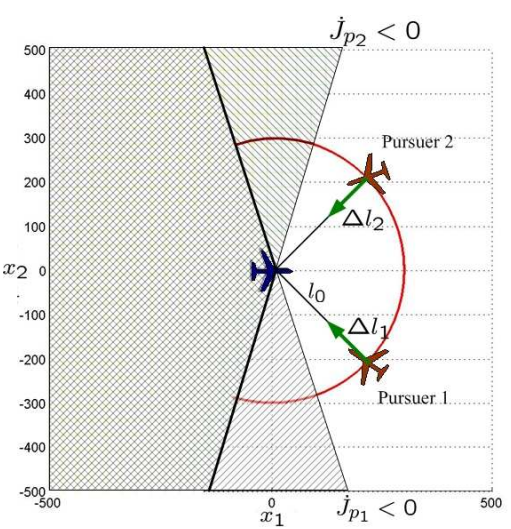

(a) Computation of the forward reachable set for each player.

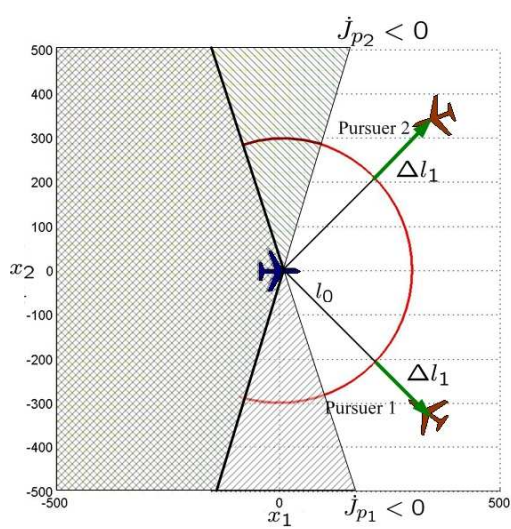

(b) Reflection of the terminal distance.

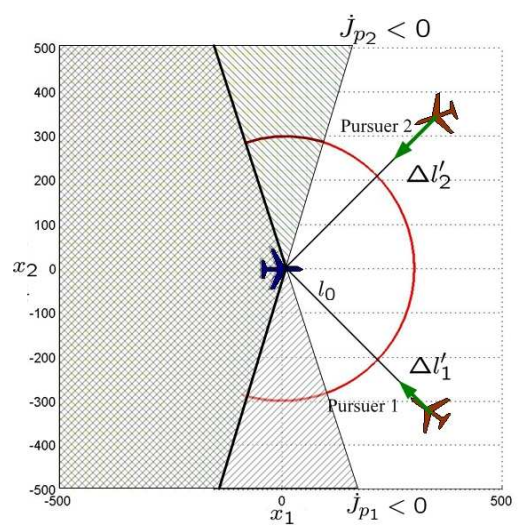

(c) Computation of the forward reachable set with a new usable part.

Figure 4. Snap shots of the reflection iteration of the forward reachable set at given initial conditions of $x_{31}=110$ and $x_{32}=-140$ degrees.

set computation. The corresponding forward reachable set $\mathcal{F}$ is given by propagating the dynamics forward in time:

$$
\mathcal{F}=\left\{\left(x_{1}, x_{2}\right) \mid \int_{t=0}^{\left.t\right|_{j(x)=0}} f\left(x, u^{*}, d^{*}\right) d t, \forall\left(x_{1}, x_{2}\right) \in \mathcal{G}_{0}\right\}
$$

where $\mathcal{G}_{0}$ is the usable part:

$$
\mathcal{G}_{0}=\{(x, y) \mid \dot{J}(x, y)<0\}
$$

and $d^{*}, u^{*}$ are defined in (9) and (10). The result is shown in Figure 3(a). This reachable set is such that if the game starts at the given initial condition of $\left(x_{1_{0}}, x_{2_{0}}, 130^{\circ}\right)$ of Figure 2(c), the separation distance between the players is guaranteed to be $l^{*}=\sqrt{x_{1_{0}}^{2}+x_{2_{0}}^{2}}-\sqrt{x_{1_{f}}^{2}+x_{2_{f}}^{2}}$ at the terminal condition (i.e., $\dot{J}=0$ ) if both players play $\left(u^{*}, d^{*}\right)$. The complete reachable set is obtained over all possible relative heading angles, $0 \leq x_{3} \leq 2 \pi$, as seen in Figure $3(\mathrm{~b})$.

The great advantage of this forward reachable set comes from the computational simplicity, which only requires solving a set of ordinary differential equations (ODEs), and from the fact that the set is only needed to determine initial conditions of the game (i.e., the set is no longer necessary once the evader applies its control strategy, and thereafter, the corresponding minimum separation distance is guaranteed). However, the drawback is that the minimum separation distance guaranteed is varied depending on initial conditions of the game.

Therefore, in order to guarantee a fixed minimum separation distance independent of initial conditions, we introduces a new concept: "reflection" of the forward reachable set. For simplicity, consider a three player game (two pursuers and one evader). The reflection of the forward reachable set is computed as follows:

- step 1: Identify the usable part, $\mathcal{G}_{0}=\left\{\left(x_{1}, x_{2}\right) \mid \dot{J}_{p_{1}}<0 \vee \dot{J}_{p_{2}}<0\right\}$, compute the corresponding reachable set for individual players (as we discussed earlier), and store $\Delta l_{1}$ and $\Delta l_{2}$ at the terminal condition, $\left(\dot{J}_{p_{1}}=0\right) \wedge\left(\dot{J}_{p_{2}}=0\right)$ (see Figure $\left.4(\mathrm{a})\right)$ :

$$
\Delta l_{i}=l_{0}-l_{i}^{*}
$$

where $l_{0}$ is the chosen minimum separation distance and $l_{i}^{*}$ is the evader's terminal separation distance from the $i$ th pursuer.

- step 2: Reflect $\max \left(\Delta l_{1}, \Delta l_{2}\right)$ outwards and recompute the usable part, $\mathcal{G}_{0}^{\prime}=\frac{l_{0}+\max \left(\Delta l_{1}, \Delta l_{2}\right)}{l_{0}} \mathcal{G}_{0}$ (see Figure 4(b)). 


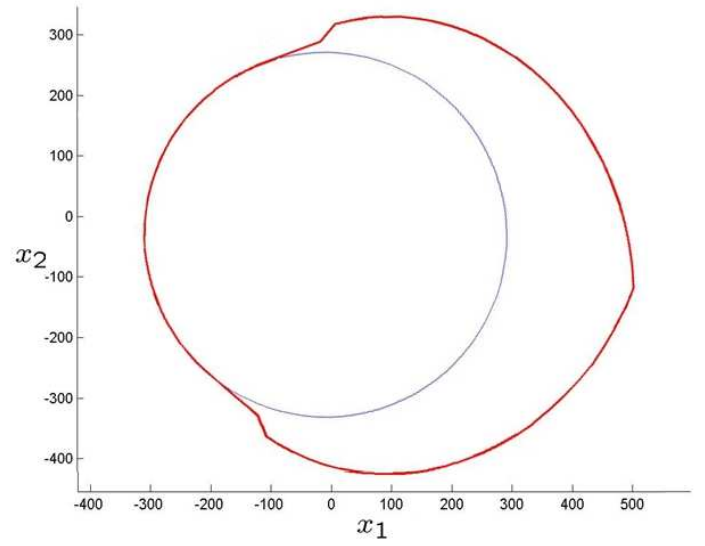

(a) A slice of the reflection of the forward reachable set at given initial conditions of relative headings, $x_{31}=110$ and $x_{32}=-140$ degrees.

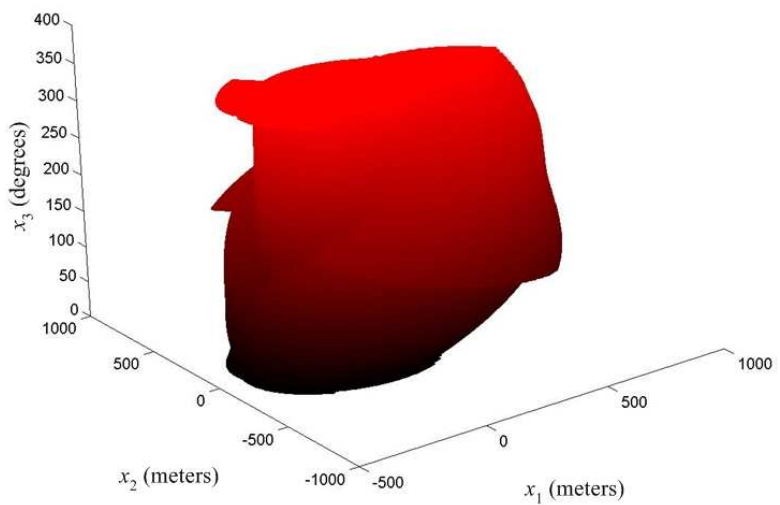

(b) The complete reflection of the forward reachable set.

Figure 5. Reflection of the Forward reachable set for a three player game.

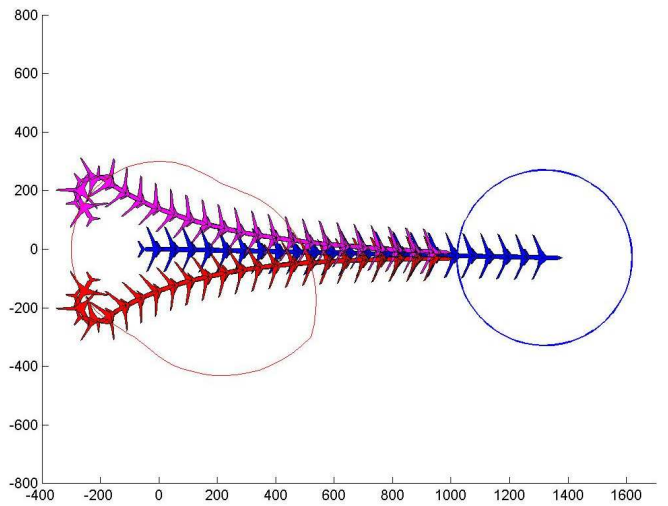

(a) Scenario 1 .

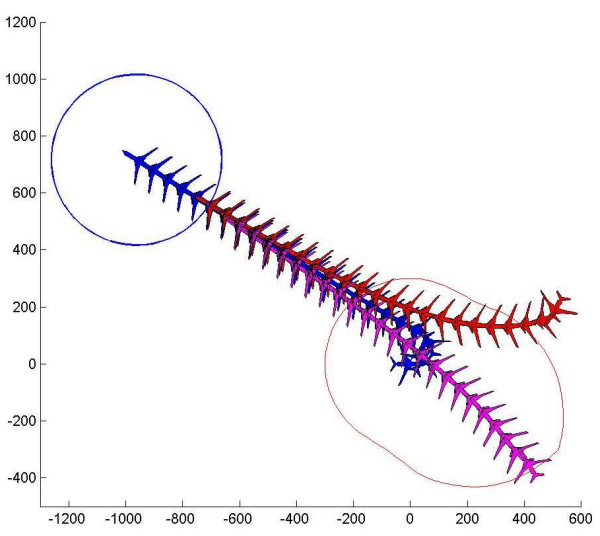

(b) Scenario 2 .

Figure 6. Trajectories of the players with the symmetry of the pursuer's starting positions

- step 3: Compute the forward reachable set with the usable part $\mathcal{G}_{0}^{\prime}$ obtained from step 2, and check whether terminal distances, $\left(l_{1}^{*}, l_{2}^{*}\right)$, with all pursuers satisfy the following condition, $\left(l_{1}^{*}>l_{0}\right) \wedge\left(l_{2}^{*}>l_{0}\right)$. If not, repeat the step 2 and 3 until this condition is satisfied (see Figure 4(c)).

The iteration process is guaranteed to converge since all players are assumed to be equally capable (the more far away from the pursuers is the evader, the higher the chance the evader has to avoid capture). The resulting reflection of the forward reachable set is shown in Figure 5(a), and the complete set in Figure 5(b).

\section{Simulation Results}

To illustrate the strategies from the previous sections, we present four representative scenarios. Figure 6(a) shows the trajectories in real $x$ - $y$ axes for the situation in which the evader is initially at the origin of the $x-y$ axes and the pursuers are initially symmetrically placed with respect to the $x$ axis. The pursuers are also positioned intentionally on the boundary of the forward reachable set so that the evader can apply its control strategy when the game begins. Due to the symmetry of the pursuer's starting position as well as their relative configuration against the evader (i.e., $\dot{J}_{p_{1}}(0)>0$ and $\left.\dot{J}_{p_{1}}(0)>0\right)$, the evader follows a

$$
6 \text { of } 10
$$


straight-line path along the $x$ axis which results in no capture by either of the pursuers at the end of the game.

Figure 6(b) shows trajectories for a different (asymmetric) initial condition of the pursuers. The evader may choose to turn toward either pursuer 1 (pink) or pursuer 2 (red). However, since pursuer 1 is on the boundary of the corresponding forward reachable set (i.e., pursuer 1 is an immediate threat against the evader), the evader turns left and follows a straight line to maintain relative distances from the pursuers. None of the pursuers were able to capture the evader since the evader's control strategy was applied when either of the pursuers (pursuer 1 in this case) first touches the corresponding forward reachable set.

Figure 7 depicts the scenario that starts from a two player game and then is switched to a three player game as the second pursuer is injected. Therefore the evader uses the control strategy and reachable set derived from the two player game first, and then uses those derived from the three player game once it detects the second pursuer.

Finally, Figure 8 depicts the scenario that starts from a two player game and then is switched to three and four player games as the second and third pursuers are injected in sequence. When pursuer 3 (yellow) comes in, it comes in with an initial condition so that the evader can no longer stay safe (pursuer 2 is already inside the corresponding reachable set (green) as seen in Figure 8(a)). Therefore, the evader was eventually captured by pursuer 2 as the game proceeded (see Figure $8(\mathrm{~b})$ and $8(\mathrm{c}))$.

\section{Pursuer's Control Strategy with Different Value Function}

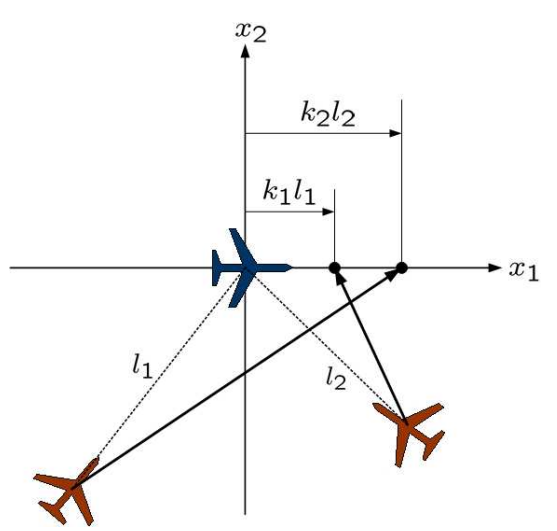

Figure 9. Configuration of the pursuers based on leading.

In the previous sections, the pursuer's strategy was derived from the simple value function of the relative distance with the evader. Obviously, using a different value function would lead a different performance measure.

In this section, we explore a different value function based on leading or predicting the evader's flight path using the current measurement of the evader's state. The idea is similar to a proportional guidance used in target tracking. Based on this, how much the pursuer can lead the evader is simply determined by how far the pursuer is from the evader in every instant, as seen in Figure 9. Therefore, the $i$ th pursuer's value function is modified as

$$
J_{p_{i}}=\left(x_{1 i}-k_{i} l_{i}\right)^{2}+x_{2 i}^{2}
$$

where $k_{i}$ is a proportional constant and $l_{i}$ is the relative distance between the $i$ th pursuer and the evader.

The resulting control strategy is similarly obtained as

$$
\begin{aligned}
d_{i}^{*} & =\min _{\left\|d_{i}\right\| \leq \nu} \ddot{J}_{p_{i}} \\
& =-\operatorname{sign}\left(-k_{i} l_{i} \sin x_{3 i}+x_{2 i} \cos x_{3 i}-x_{1 i} \sin x_{3 i}\right) \nu
\end{aligned}
$$

The above control strategy is compared with one used in the previous simulations, and the result is shown in Figure 10, Figure 10(a) shows trajectories of the players using the original control strategies and Figure 10(b) using (15). The initial condition and the evader's control strategy are the same for both simulations. For evaluation of the pursuer's control strategy, the reachable set was not used (i.e., all players immediately apply their strategies when the game starts). It is seen that the pursuers with the new strategy capture the evader eventually. The resulting motions can be described as follows. Pursuer 1 in the upper left corner initially does not follow the evader since the evader's projected capture point is on his way; instead pursuer 1 loiters around this point. Meantime, pursuer 2 in the lower right corner follows the evader. As the evader is getting closer to pursuer 1, the capture point projected by pursuer 1 aims toward the evader itself. Therefore, after a certain time, pursuer 1 starts to follow the evader, and this forces the evader to take another evasive motion which results in turning toward a direction where it might maintain relative distances with the pursuers. However, by the time this happens, both pursuers take advantage of the situation and eventually capture the evader. 


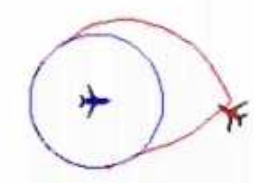

(a) Initial configuration of a two player game.

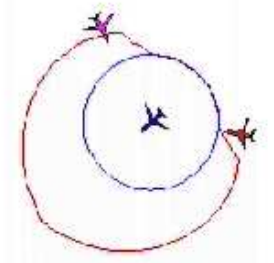

(d) The evader takes another evasive action when the second pursuer touches the corresponding reachable set.

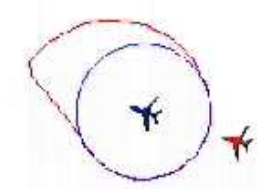

(b) The evader takes an evasive action when the pursuer touches the reachable set.

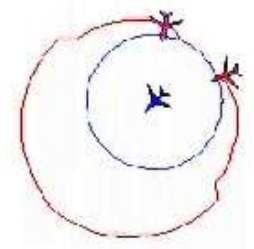

(e) The evader follows a straight line to maintain relative distances with the pursuers.

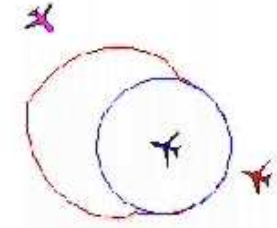

(c) The second pursuer is injected and the evader changes the mode to a three player game to accommodate the second one.

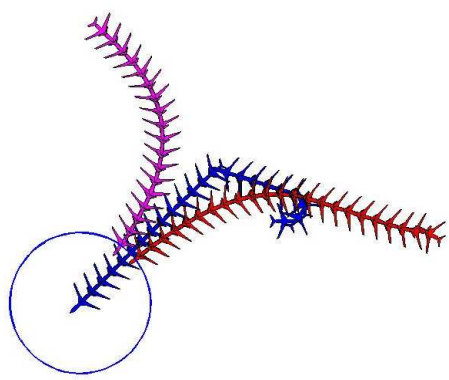

(f) Trajectories of the players during the game.

Figure 7. Snap shots of two and three player games and the corresponding trajectories.

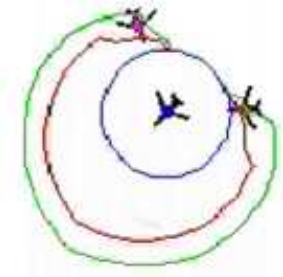

\#

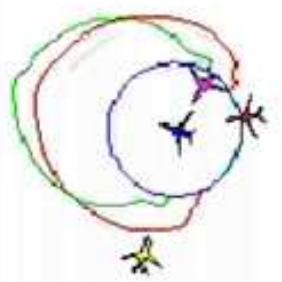

(b) The evader immediately takes an evasive action, but pursuer 2 (top) is already inside the reachable set. (a) Pursuer 2 (yellow) is injected from Figure $7(\mathrm{~d})$, and the resulting reachable set (green) shows that the evader is no longer is safe.

Figure 8. Snap shots of two, three, and four player games.

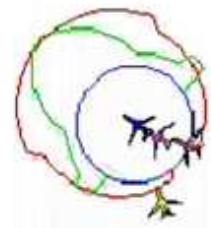

(c) The game continues, but capture is occurred by pursuer 2 .

\section{8 of 10}




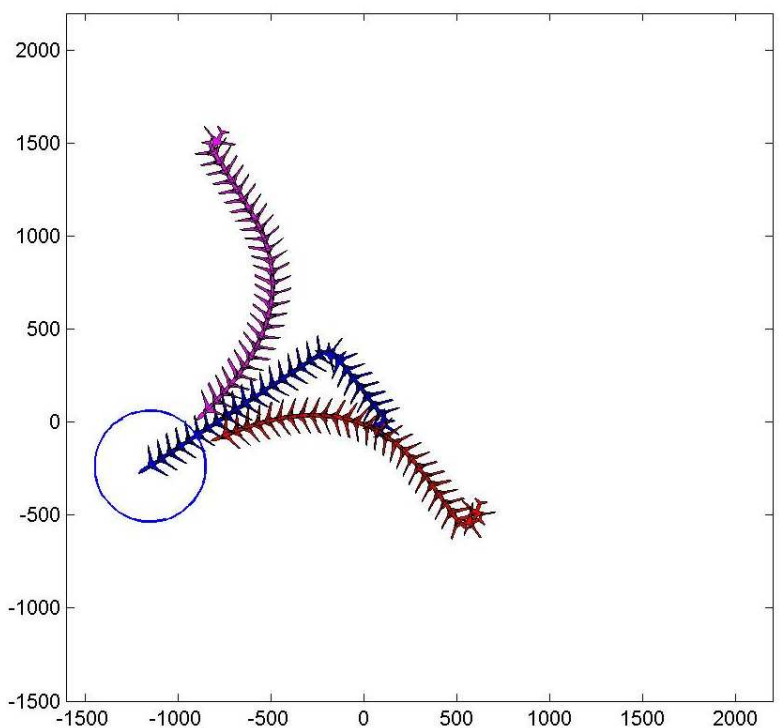

(a)

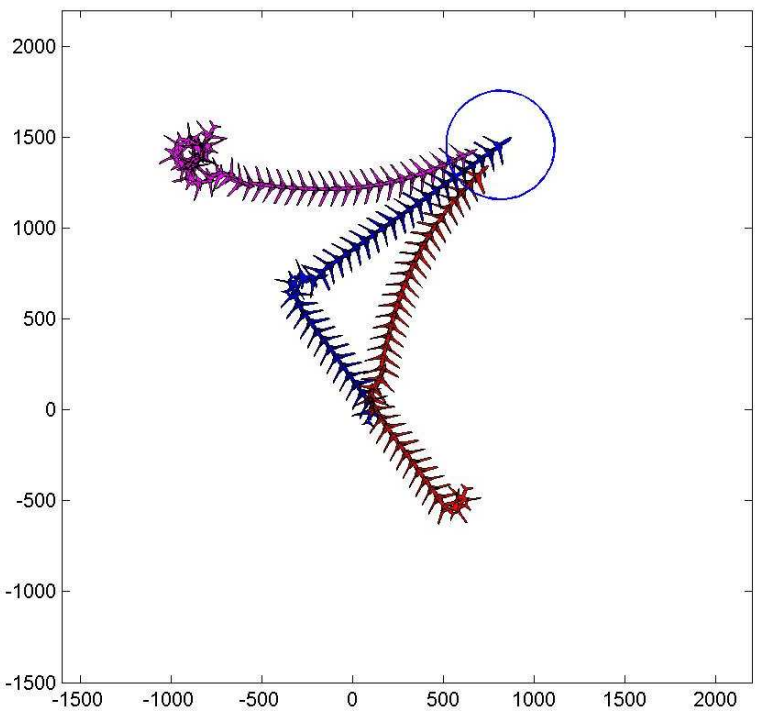

(b)

Figure 10. Comparison of the pursuer's control strategies derived from different value functions.

\section{Conclusion}

This paper presents a new methodology for multi-player pursuit and evasion game. The proposed control strategies (which are not necessarily optimal) were derived from the direct differentiation of chosen value functions, ensuring safety yet allowing use for practical implementations on real-time control systems for autonomous vehicles. In order to guarantee "minimum separation distance", a fast computation of reachable sets which partitions "safe" from "unsafe", or "capture" from "evade" are developed. As opposed to Hamilton Jacobi Isaacs equations whose solution is obtained by solving a set of partial differential equation, the proposed algorithm requires a set of ordinary differential equations to obtain the corresponding solution. Moreover, this solution is only needed to determine initial conditions for the evader to apply the chosen control strategy: once the control is applied, it is guaranteed to be safe thereafter.

Since the strategies includes no explicit cooperation among pursuers (i.e., individual pursuers are trying to minimize their value function only), we are currently studying a cooperative strategy where individual pursuers take into account control strategies of others in order to maximize the collective benefits.

\section{References}

${ }^{1}$ Stipanović, D., Inalhan, G., Teo, R., and Tomlin, C.J., "Decentralized Overlapping Control of a Formation of Unmanned Aerial Vehicles," To appear, Automatica.

${ }^{2}$ Tomlin, C.J., P. G. and Sastry, S., "Conflict Resolution for Air Traffic Management: A Study in Multi-Agent Hybrid Systems," IEEE Transactions on Automatic Control, Vol. 43, No. 4, April 1998.

${ }^{3}$ Cremean, L., Dunbar, W.B., van Gogh, D., Hickey, J., Klavins, E., Meltzer, J., and Murray, R.M., "The Caltech MultiVehicle Wireless Testbed," Nevada, December 2002.

${ }^{4}$ Richards, A., Bellingham, J., Tillerson, M., and How, J.P., "Co-ordination and Control of Multiple UAVs," Proceedings of AIAA Guidance, Navigation, and Control Conference, August.

${ }^{5}$ Shim, D.H., Kim, H.J., Sastry, S., "Decentralized nonlinear model predictive control of multiple flying robots," Proceedings. 42nd IEEE Conference on Decision and Control, December 2003.

${ }^{6}$ Isaacs, R., Differential games, New York:Wiley, 1965.

${ }^{7}$ Merz, A., "The Game of Two Identical Cars," Journal of Optimization Theory and Applications, Vol. 9, No. 5, 1972, pp. 324-343.

${ }^{8}$ Mitchell, I., Bayen, A.M., and Tomlin, C.J., "Computing Rechable Sets for Continuous Dynamic Games Using Level Set Methods," submitted to IEEE Transactions on Automatic Control.

${ }^{9}$ Teo, R. and Tomin, C., "Computing Danger Zones for Provably Safe Closely Spaced Parallel Approaches," AIAA Journal of Guidance, Control, and Dynamics, Vol. 26, No. 3, May-June 2003. 
${ }^{10}$ Pashkov, A.G. and Terekhov, S.D., "A Differential Game of Approach with Two Pursuers and One Evader," Journal of Optimization Theory and Applocations, Vol. 55, 1987.

${ }^{11}$ Star, A.W. and Ho, Y.C., "Further Properties of Nonzero-Sum Differential Games," Journal of Optimization Theory and Applications, Vol. 3, No. 4, 1969.

${ }^{12}$ Vaisbord, E.M. and Zhukovsky V.I., Introduction to Multi-Player Differential Games and Their Applications, New York:Gordon and Breach, 1988.

${ }^{13}$ Mitchell, I. and Tomlin, C., "Overapproximating Reachable Sets by Hamilton-Jacobi Projections," Journal of Scientific Computation, Vol. 19, No. 1, December 2003.

${ }^{14}$ Stipanović, D., Hwang, I. and Tomlin, C.J., "Computation of an Overapproximation of the Backwards reachable set using Subsystem Level Set Functions," Dynamics of Continuous, Discrete, and Impulsive Systems,Series A: Mathematical Analysis 11, 2004, pp. 399-411.

${ }^{15}$ Crandall, M.G. and Breakwell, J.V., "Two approximation Schemes for Hamilton Jacobi Equations," Mathematics and Computation, Vol. 43, No. 167, 1984.

${ }^{16}$ Kurzhanski, A. and Valyi, A., Ellipsoidal Calculus for Estimation and Control, Birkhauser, 1997.

${ }^{17}$ Kurzhanski, A. and Varaiya, P., "Reachability analysis for uncertain systems-the ellipsoidal technique," Dynamics of Continuous, Discrete Impulsive Systems Series 5: Applications Algorithms, Vol. 9, No. 3), 2002.

${ }^{18}$ Stipanović D., Shankaran, S. and Tomlin, C.J., "Strategies for Agents in Multi-Player Pursuit-Evasion Games," Proceedings of 11th International Symposium on Dynamic Games and Applications, Arizona, December 2004. 